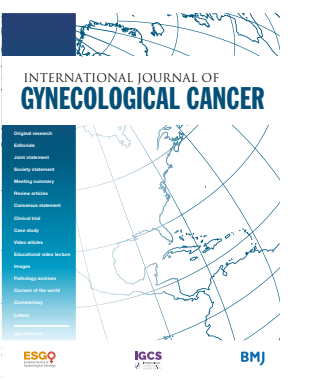

\title{
Ultrasound, macroscopic and histological features of endometrioid and clear cell ovarian cancer
}

\author{
Giulia Bolomini, Valentina Bertoldo, Gian Franco Zannoni, Federica Pozzati
}

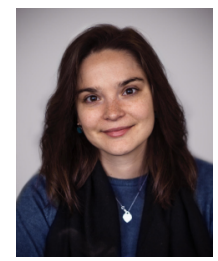

\section{Giulia Bolomini}

${ }^{1}$ Dipartimento Scienze della Salute della Donna, del Bambino e di Sanità Pubblica, Fondazione Policlinico Universitario A Gemelli, IRCCS, Rome, Italy

Biography: Dr. Giulia Bolomini is a gynecologist with ultrasound expertise. She works in the Gynecology Oncology department at the Agostino Gemelli University Hospital, IRCCS in Rome.
Dipartimento Scienze della Salute della Donna, del Bambino e di Sanità Pubblica, Fondazione Policlinico Universitario A Gemelli, IRCCS, Rome, Italy

\section{Correspondence to}

Dr Giulia Bolomini, Dipartimento Scienze della Salute della Donna, del Bambino e di Sanità Pubblica, Fondazione Policlinico Universitario A. Gemelli, IRCCS, Rome, Italy; giulia.bolomini@ gmail.com

Accepted 21 September 2020 Published Online First

2 November 2020

\section{SUMMARY}

The objective of Video 1 is to present two cases of epithelial ovarian carcinomas, examined at the Gynecologic Oncology Unit of the Fondazione Policlinico Universitario Agostino Gemelli, IRCCS, in Rome, Italy. Our local Ethics Committee was consulted, but this article is exempt from the need for approval.

The first case is a 45 years' old woman with no family history of cancer, and a past medical history of hysterectomy and bilateral salpingectomy performed three years before because of diffuse uterine fibromatosis, and her history was negative for endometriosis. The patient was referred to our center for bilateral adnexal masses incidentally detected during a routine transvaginal ultrasound examination performed at another hospital. Serum levels of oncological markers were: CA $19.983 .2 \mathrm{U} / \mathrm{mL}$ (reference range: $0-37 \mathrm{U} /$ $\mathrm{mL}$ ), CA $12551.4 \mathrm{U} / \mathrm{mL}$ (reference range: $0-35 \mathrm{U} /$ $\mathrm{mL}$ ), CA $15.319 .3 \mathrm{U} / \mathrm{mL}$ (reference range: $0-32.5 \mathrm{U} /$ $\mathrm{mL}$ ), AFP $2.70 \mathrm{ng} / \mathrm{mL}$ (reference range: $0-6 \mathrm{ng} / \mathrm{mL}$ ), and CEA $4.3 \mathrm{ng} / \mathrm{mL}$ (reference range: $0-5 \mathrm{ng} / \mathrm{mL}$ ). Transvaginal ultrasound examination performed at our center showed a left multilocular solid tumor of $69 \times 46 \times 46 \mathrm{~mm}$ in size, with low-level content and a large central solid component of $37 \times 30 \times 32 \mathrm{~mm}$ in size. The solid component was entrapped within locules that gave the tumor a cockade-like appearance (Figure 1). ${ }^{1} \mathrm{~A}$ right multilocular solid tumor of $55 \times 27 \times 35 \mathrm{~mm}$ in size was also seen and a cockade sign was also observed in the right ovarian cyst. At
Check for updates

(C) IGCS and ESGO 2021. No commercial re-use. See rights and permissions. Published by BMJ.

\begin{tabular}{l} 
To cite: Bolomini G, \\
Bertoldo V, Zannoni GF, \\
et al. Int J Gynecol Cancer \\
2021;31:938-939. \\
\hline
\end{tabular}
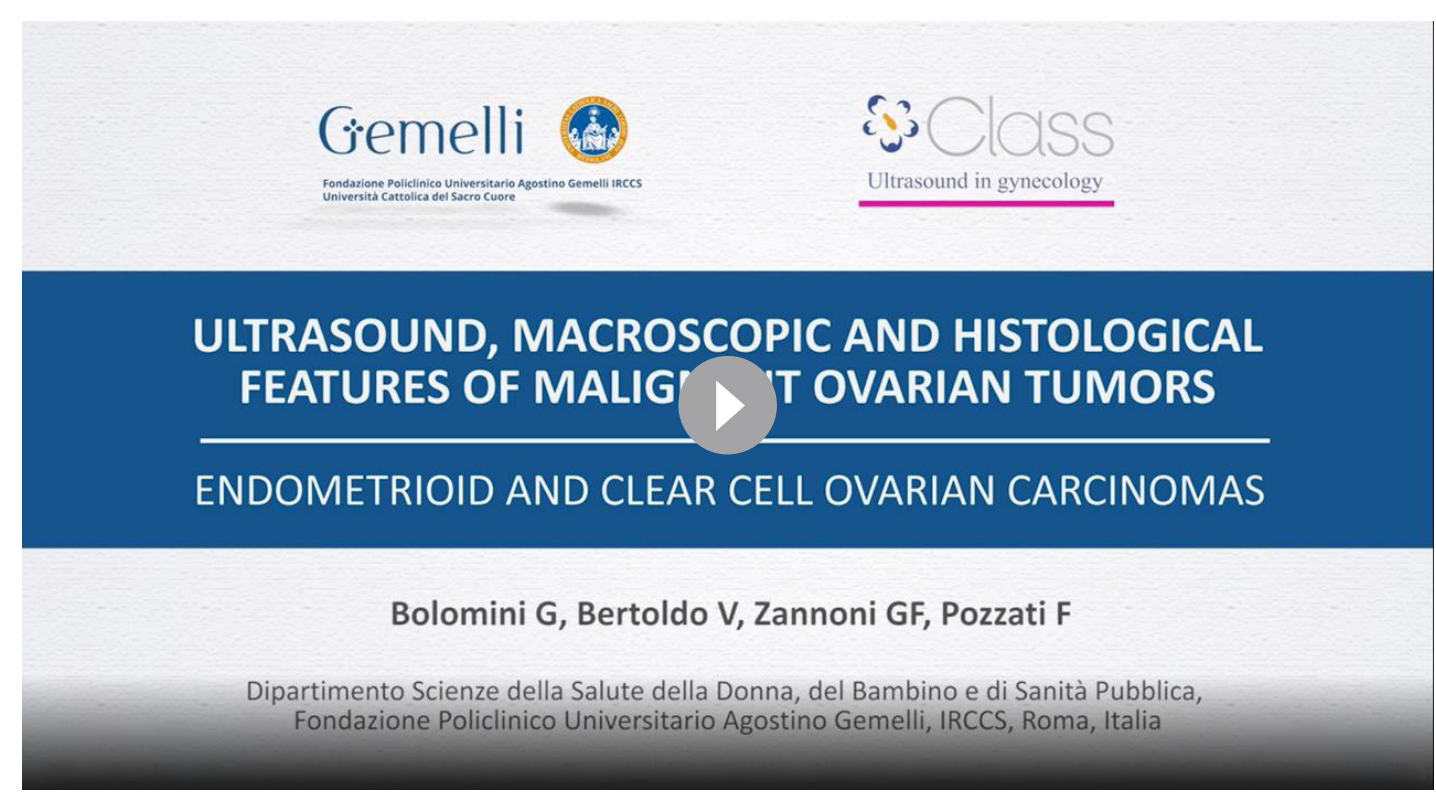

\section{ULTRASOUND, MACROSCOPIC AND HISTOLOGICAL FEATURES OF MALIG $>$ T OVARIAN TUMORS}

ENDOMETRIOID AND CLEAR CELL OVARIAN CARCINOMAS

Video 1 Ultrasound, macroscopic and histological features of endometrioid and clear cell ovarian cancer 




Figure 1 Endometrioid ovarian carcinoma not developing from endometriosis appears as a multilocular-solid lesion with a large central solid component entrapped within locules, giving the tumor a cockade-like appearance.

color doppler examination, both ovarian masses showed a rich vascularization. We applied the IOTA ADNEX model ${ }^{2}$ on the biggest multilocular solid mass, that is, the left ovarian lesion. The IOTA ADNEX model showed an increased risk of malignancy, with highest relative risk for either borderline ovarian tumor or stage I ovarian cancer (link to the IOTA ADNEX model calculator: https://www. iotagroup.org/sites/default/files/adnexmodel/IOTA20-20ADNEX20model.html). Moreover, the tumor was classified as 0-RADS 5 . $^{3}$

At laparoscopy, bilateral masses were visualized. Intraoperative frozen section of both ovarian masses were positive for adenocarcinoma. Therefore, bilateral oophorectomy, omentectomy, bilateral pelvic lymphadenectomy, and para-aortic lymphadenectomy were performed. There was no residual tumor. At macroscopic examination, multilocular solid-cystic masses were described. ${ }^{4}$ At histology, adenocarcinoma was confirmed within the endometriotic cyst and final histology report was positive for endometrioid ovarian carcinoma, G2, Figo stage IB $2 .^{5}$

The second case is a 54 years' old woman with a family history of endometrial cancer (mother) and cervical cancer (sister). The patient was referred to our center for a pelvic mass of $70 \mathrm{~mm}$ in size detected during an ultrasound examination performed at another hospital for abdominal tenderness. Serum levels of oncological markers were: CA $125211 \mathrm{U} / \mathrm{mL}$ (reference range: 0-35U/ $\mathrm{mL}$ ), CA15.3 79 U/mL (reference range: $0-32.5 \mathrm{U} / \mathrm{mL}$ ), and CA 19.9 $179 \mathrm{U} / \mathrm{mL}$ (reference range: $0-37 \mathrm{U} / \mathrm{mL}$ ).

Transvaginal ultrasound examination performed at our center showed a left multilocular solid tumor ( $<10$ locules) of $67 \times 66 \times 84 \mathrm{~mm}$ in size, with low-level content, irregular internal walls for the presence of multiple papillary projections, and a solid component of $39 \times 21 \mathrm{~mm}$ in size. ${ }^{6}$ The mass showed a moderate vascularization at color Doppler examination. The IOTA ADNEX model $^{2}$ showed an increased risk of malignancy, with highest relative risk for stage II-IV ovarian cancer (link to the IOTA ADNEX model calculator: https://www.iotagroup.org/sites/default/files/ adnexmodel/IOTA20-20ADNEX20model.html). Moreover, the tumor was classified as 0-RADS $5 .^{3}$
Laparoscopy confirmed the presence of a left adnexal mass. Intraoperative frozen section of the left ovarian mass was positive for adenocarcinoma. Therefore, extrafascial simple total hysterectomy, bilateral salpingoophorectomy, para-aortic lymphadenectomy, removal of the left external iliac lymph nodes, and infracolic omentectomy were performed. The macroscopic assessment of the right mass confirmed the presence of a multilocular solid cystic tumor with pale yellow nodules. ${ }^{7}$ The final histology report was positive for ovarian clear cell carcinoma FIGO stage IIIA. ${ }^{8}$

Acknowledgements We thank Prof. Antonia Carla Testa, Prof. Giovanni Scambia, and Dr. Francesca Moro, Dipartimento Scienze della Salute della Donna, del Bambino e di Sanità Pubblica, Fondazione Policlinico Universitario Agostino Gemelli, IRCCS, Roma, Italy for the scientific supervision. We also thank Dr. Paola Romeo, Dr. Valeria Verdecchia, and Mr. Emerson Marinho Pinto, Dipartimento Scienze della Salute della Donna, del Bambino e di Sanità Pubblica, Fondazione Policlinico Universitario Agostino Gemelli, IRCCS, Roma, Italy for the realization of the video.

Contributors All the authors took part in planning, conducting, and reporting the work.

Funding The authors have not declared a specific grant for this research from any funding agency in the public, commercial, or not-for-profit sectors.

Competing interests None declared.

Patient consent for publication Not required.

Provenance and peer review Not commissioned; externally peer reviewed.

ORCID iD

Federica Pozzati http://orcid.org/0000-0003-3503-2451

\section{REFERENCES}

1 Moro F, Magoga G, Pasciuto T, et al. Imaging in gynecological disease (13): clinical and ultrasound characteristics of endometrioid ovarian cancer. Ultrasound Obstet Gynecol 2018;52:535-43.

2 Van Calster B, Van Hoorde K, Valentin L, et al. Evaluating the risk of ovarian cancer before surgery using the ADNEX model to differentiate between benign, borderline, early and advanced stage invasive, and secondary metastatic tumours: prospective multicentre diagnostic study. BMJ 2014;349:g5920.

3 Andreotti RF, Timmerman D, Strachowski LM, et al. O-RADS US risk stratification and management system: a consensus guideline from the ACR Ovarian-Adnexal reporting and data system committee. Radiology 2020;294:168-85.

4 Longacre TA, Wells M. Endometrioid Tumors. In: Kurman RJ, Hedrick Ellenson L, Ronnet BM, eds. Blaustein's Pathology of the Female Genital Tract. New York, NY, USA; Dodrecht Heidelberg: London, UK: Springer, 2011: 748-58.

5 Longacre TA, Wells M. Endometrioid tumours. In: Kurman RJ, Carcangiu ML, Herrington CS, et al, eds. WHO classification of tumours of female reproductive organs. Lyon, France: IARC, 2014: 29-32.

6 Pozzati F, Moro F, Pasciuto T, et al. Imaging in gynecological disease (14): clinical and ultrasound characteristics of ovarian clear cell carcinoma. Ultrasound Obstet Gynecol 2018;52:792-800.

7 Longacre TA, Wells M. Serous tumors. In: Kurman RJ, Carcangiu ML, Herrington CS, et al, eds. WHO classification of tumours of female reproductive organs. Lyon, France: IARC Press, 2014: 15-24.

8 Seidman JD, Cho KR. Surface epithelial tumors of the ovary. In: Kurman RJ, Ellenson LH, Ronnet BM, eds. Blaustein's Pathology of the Female Genital Tract. 6th edn. Boston, MA: Springer, 2011: 679-784. 